\title{
Chemical Degradation of Epoxy-Polyamide Primer by Electrochemical Impedance Spectroscopy
}

\author{
Ramesh Duraisamy, Kannan Pownsamy, and Ghebray Asgedom \\ Department of Chemistry, College of Science, Eritrea Institute of Technology, Mai Nefhi, Asmara, Eritrea \\ Correspondence should be addressed to Ramesh Duraisamy, drrameshmcas@gmail.com
}

Received 24 February 2012; Accepted 10 April 2012

Academic Editors: K. N. Allahar, G. Bereket, C. V. D’Alkaine, and R. Salghi

\begin{abstract}
Copyright (C) 2012 Ramesh Duraisamy et al. This is an open access article distributed under the Creative Commons Attribution License, which permits unrestricted use, distribution, and reproduction in any medium, provided the original work is properly cited.
\end{abstract}

\begin{abstract}
The degradation of organic-inorganic hybrid materials based on epoxy resin was characterized electrochemically in aggressive chemical electrolyte. In the present study, the hybrid material as primer was prepared from epoxy resin pigmented by zinc phosphate cured with polyamide (EPZ). The hybrid material was coated on mild steel substrate, and the corrosion behavior was studied by electrode-potential time measurements and mainly by electrochemical impedance spectroscopy (EIS) in $5 \% \mathrm{NaCl}$ solution. The impedance parameters, namely, coating capacitance $\left(C_{c}\right)$, pore resistance $\left(R_{\mathrm{po}}\right)$, charge transfer resistance $\left(R_{t}\right)$, double layer capacitance $\left(C_{\mathrm{d} 1}\right)$, and break point frequency $\left(f_{b}\right)$, corresponding to $45^{\circ}$ phase angle as a function of time of exposure were estimated. The observed impedance behavior were compared with the established equivalent electrical circuit represents the coated metal/electrolyte interface. Changes in the values of the circuit components given the information on the stages of degradation and physical phenomenon occurring throughout the degradation of primer coating were also been predicted. In addition, information related to the porous nature of the primer, limited passivation effect, and delamination of coating with longer exposure that resulted in the diffusion controlled corrosion of metal are also recognized. Thus, results indicate that the EPZ coating had good corrosion resistance. This could be a nonpolluting alternative to the traditional chromate like environmentally harmful coatings.
\end{abstract}

\section{Introduction}

Epoxy coatings, generally crosslinked with amines or polyamides, are widely used as heavy duty moisture and chemical resistance, well adhesion, and corrosion-resistive coatings and lining in various environments, since they create a three dimensional protective network [1]. The mechanism by which a polymer coating protects a metal substrate against corrosion is of greater interest. The coating serves as a mass transport barrier to the substrate, but this role does not describe completely the phenomenona that are observed in some systems. Though epoxy resins have been widely described for various coating applications with progress of time, water, oxygen, and ions penetrate the coating, which initiates corrosion at metal-coating interface $[2,3]$. This penetration of species may occur either by diffusion through the polymer or more likely by ingress at local defects. Pigments can be added to organic coatings in order to improve their protection capabilities. The pigments might protect by physicochemical (barrier mechanism), electrochemical, or ion-exchange mechanism [4]. Anticorrosive primers are generally applied directly on metal substrate to protect against corrosion as a second line of defense. Thus the study of anticorrosive behavior of primer is essential to know about the changes taking place in the paint film and corrosion reaction at metal-coating interface.

In this work, a commercial zinc phosphate was incorporated into epoxy-polyamide matrix in order to evaluate the contribution of this pigment to the protection properties of an epoxy-polyamide coating. Zinc phosphate is a well-known pigment that shows anticorrosion properties by forming a protective layer when in contact with iron ions of mild steel. In addition the zinc phosphate composite films to act as a protective coating for mild steel are feasible and might be advantageous in the replacing of the conventional system of phosphatized layers on mild steel that was recognized by many researchers [5-8]. Besides, conversion treatments such as phosphatization and chromatization face restrictions 
due to their toxicity and due to environmental concerns. Improving the homogeneity of pigment distribution within the epoxy-polyamide film and its surface would probably enhance its protective action.

Maintain a good coating performance; it is very important to detect the performance of coatings in corrosion environments [9]. Electrochemical impedance spectroscopy (EIS) has established itself as a primary method of evaluating the performance and degradation process of organic coating systems [10-14]. Its advantages over conventional DC techniques are well documented [5-11]. By analyzing the impedance spectra, some important parameters might be obtained such as coating resistance, coating capacitance, electrochemical reaction resistance, and double-layer capacitance, which provide the evaluation of the coating systems $[15,16]$. The high-frequency impedance behavior represents the coating characteristics and that of low-frequency part of impedance represents the corrosion reactions occurring at the bottom of the pores of the coating [17-19]. Generally, the coatings with resistance over $10^{8} \Omega \mathrm{cm}^{2}$ provide good corrosion protection, while those with resistance under $10^{6} \Omega \mathrm{cm}^{2}$ provide poor corrosion protection [20]. Macedo et al. [21] recently studied the performance of epoxy, alkyd, and polyurethane paints by EIS, pointing out that although EIS is an excellent tool to monitor the behavior of organic coatings, a deeper understanding of the electrolyte/paint film interactions is absolutely essential to perfectly analyze the impedance data. In addition, because the relatively long testing time and the complicated spectrum analysis, EIS methods are mainly applied in laboratory studies. Some authors studied fast evaluation methods for coating performance by EIS, trying to find the parameters which reflected the coating performance and can be obtained quickly, in order to avoid the complicated spectrum analysis.

EIS has also been used to study the performance of thick coatings with or without pigments and to elucidate degradation mechanism either by studies about defective areas in coatings or studying the anodic and cathodic sites separately [22-26]. From these investigations useful information has been obtained for the formation of corrosion under a defective coating on the diffusion limitations and on the pathways for ionic conduction between anodic and cathodic sites. In spite of large number of articles published on the use of EIS to characterize organic coatings, measurement of setup and analyzing the procedure still needs further optimization of its wide acceptance by manufactures and users.

EIS analysis and prediction of long-term behavior of translucent pigmented and unpigmented epoxy and conventional epoxy-polyamide system with varying coating thickness have been reported by Scully [22]. The pigments used were mostly quartz to study its effect on the deterioration of coating using Bode plots, open-circuit potential, and lowfrequency impedance. The anticorrosion behavior of different inorganic pigments in epoxy-polyamide [3], degradation of epoxy coating with artificial defect [27, 28], and chemical degradation of novolac coatings [29] has been analyzed by EIS to obtain information on the corrosion protection. Epoxies represent perhaps the best combination of corrosion resistance and mechanical properties, when they
TABLE 1: Composition of zinc phosphate containing epoxy-polyamide hybrid primer.

\begin{tabular}{lc}
\hline Composition & Amount $(\% \mathrm{wt})$ \\
\hline PACK A (for $100 \mathrm{gm})$ & 34.60 \\
$\quad$ Epoxy resin & 5.70 \\
Red oxide of iron & 17.15 \\
Zinc phosphate & 10.75 \\
Silica/asbestine & 20.25 \\
Toluene, MIBK, and butanol (in ratio & \\
of $(1: 1: 1)$ ) & 11.55 \\
\hline PACK B & \\
$\quad$ Polyamide & \\
\hline
\end{tabular}

are crosslinked notably with suitable amines or polyamides $[1,6]$. The coating characteristics are modified in the presence of pigments, fillers, and solvent.

Thus, the study of such coatings in terms of their impedance response can provide better insight into the mechanism of corrosion protection and can be useful in the prediction of long-term protection behavior. In the present study, the EIS measurement was carried out on steel coated with zinc phosphate-pigmented epoxy-polyamide primer (EPZ) in $5 \% \mathrm{NaCl}$ in the frequency range $100 \mathrm{KHz}$ to $10 \mathrm{~Hz}$. The impedance data over 100 days of exposure has been analyzed in terms of both Nyquist and Bode plots. In addition to the EIS studies, EPZ coating was also evaluated by potential-time measurement over 100 days of exposure. The time dependence of $C_{c}, C_{\mathrm{dl}}, R_{\mathrm{po}}, R_{t}, f_{b}$, and $E_{\mathrm{oc}}$ has been estimated to show a relationship with the observed degradation of coating.

\section{Experimental Details}

2.1. Preparation of Steel Surfaces. Cold rolled mild steel having the composition Carbon: 0.05\%, Phosphorous: $0.025 \%$, Sulphur: $0.14 \%$, Silicon: $0.03 \%$, Manganese: $0.013 \%$, and rest of iron was selected for the study. The dimensions of specimens $150 \mathrm{~mm} \times 100 \mathrm{~mm} \times 2 \mathrm{~mm}$ were cut from the rod after making into slices and adopted the posttreatment, and evaluations were off with AR-grade chemicals. The posttreated specimens were mechanically polished with 4/0 emery paper successively and dipped in $5 \% \mathrm{NaOH}$ solution for $2 \mathrm{~min}$ to activate the surface [30]. Activated specimens were cleaned with cleaning powder to remove the black colored smudge formed over the surface and were washed thoroughly with running water and dipped in conc. $\mathrm{HNO}_{3}$ solution for 60 seconds. The specimens were then washed with distilled water and used for coating of epoxy hybrid protective material. The panels were degreased with sulphur free toluene and dried before application of paint.

2.2. Preparation of Epoxy Hybrid Material and Coated Specimens. The composition of the epoxy-polyamide containing zinc phosphate is given in Table 1. The zinc phosphate-pigmented epoxy consists of one pack, and the other pack consists of polyamide curing agent. The ratio of the epoxy 
in pack A to polyamide as curing agent in pack B was kept as $3: 1$. Both packs were mixed and stirred to uniform consistency and made a hybrid-coating material called zinc phosphate epoxy-polyamide (EPZ). Two coats of EPZ were applied on activated mild steel specimen by conventional spraying. The second coat was applied after 24 hours of application of the first coat. The specimens having an average film thickness of $70 \pm 5 \mu \mathrm{m}$ were measured using Elcometer thickness meter and were selected for exposure into the aggressive aerated $5 \% \mathrm{NaCl}$ solution.

\subsection{Evaluation of Corrosion-Resistant Property. The EPZ-} coated specimens were made as working electrode over which specially arranged cylindrical glass tube of $7 \mathrm{~cm}$ length was fixed with epoxy adhesive leaving an exposed area of $1 \mathrm{~cm}^{2}$ reported [31]. The cell was filled with $5 \% \mathrm{NaCl}$ solution up to $5 \mathrm{~cm}$ length of glass tube; addition of demineralized water made the evaporation loss of electrolyte. Pretreated followed by EPZ-coated mild steel was used as working electrode in the conventional three electrode assembly having platinum foil as counter electrode and saturated calomel electrode (SCE) as reference electrode.

Corrosion resistance property of EPZ-coated specimens was evaluated through electrochemical impedance spectroscopy (EIS) and potential-time as open-circuit potential $\left(E_{\mathrm{oc}}\right)$ measurements in aerated $5 \% \mathrm{NaCl}$. The EIS measurement was carried out by using EG \& G PAR model 638 impedance measurement systems, which consisted of model 173 potentiostat, model 6310 lock-in analyzer. The EIS measurement was carried out at the impressed open-circuit potential in aqueous $5 \% \mathrm{NaCl}$ solution with 15 -minute interval to reach steady-state potential for each measurement. The frequency range of each measurement was varied from $100 \mathrm{kHz}$ to $10 \mathrm{~Hz}$ using an AC excitation potential of $10 \mathrm{mV}$. The analysis of the impedance spectra was done by fitting the experimental results to equivalent circuits using the nonlinear least square fitting Z-view software from Scribner Associates. The quality of fitting to equivalent circuit was judged firstly by the chi-square value $\left(\chi^{2}\right.$, that is, the sum of the square of the differences between theoretical and experimental points) and secondly by limiting the relative error in the value of each element in the equivalent circuit to 5\% [32]. All impedance measurements were carried out by triplicate in a Faraday cage in order to minimize external interference on the system studied.

Change in potential of EPZ-coated specimens was noticed during different time intervals over 100 days of exposure in $5 \% \mathrm{NaCl}$ under open-air atmosphere. Electronic multimeter Philips model PM 2518 was employed to monitor the potential change against saturated calomel electrode (SCE). This measurement was taken to discuss about the correlation between the corrosion potential of the coating, and real behavior of the system was documented in the literature $[29,33]$.

\section{Results and Discussion}

Impedance behavior of mild steel coated with EPZ was studied about 100 days of exposure in $5 \% \mathrm{NaCl}$ in open-air atmosphere. The results were analyzed in terms of both the Nyquist and Bode plots. From the shape of the impedance plots, three stages of degradation were identified, that is, the stage between $0-5$ days, $10-30$ days, and beyond up to 100 days. The first stage shows one time constant, the second two-time constants, and the third a predominantly diffusion controlled process. So the results are analyzed taking into consideration the representative curves of each stage. In case of one semicircle in the Nyquist plot, the $R_{\mathrm{po}}$ has been estimated from its diameter. With two semicircles, the diameter of the high-frequency semicircle represented $R_{\text {po }}$ and that of lower frequency $R_{t}$. The coating capacitance $\left(C_{c}\right)$ was estimated from the $F_{\max }$, frequency maxima of the semicircle of the Nyquist plot at which reactive component reaches a maximum and using the relationship [34]:

$$
C_{c}=\frac{1}{2 \pi\left(F_{\max } \cdot R_{\mathrm{po}}\right)} .
$$

3.1. Stage I: one Time Constant Process. Evolutions of the electrochemical behavior of epoxy-polyamide-coated specimens were clearly indicated by the EIS. In the first hours after immersion, the impedance diagram is highly capacitive, exhibiting the phase shift of $90^{\circ}$ over wide frequencies. The Nyquist and Bode plots of EPZ-coated steel in $5 \% \mathrm{NaCl}$ for selected period are shown in Figure 1. A semicircular plot corresponding to one time constant is seen between 1 hour and 5 days (Figure 1(a)) which represents an equivalent circuit as in Figure 2(a) evident from earlier [35]. The resistance $R_{\Omega}$ is the resistance of the electrolyte. It was accepted [36] that the value of coating resistance, $R_{\mathrm{ct}}$, is the best for the measurement of coating degradation, where it can be found from semicircle diameter of the Nyquist plot and shows very high capacitive and resistive values. The Bode magnitude (Figure 1(b)) plot also reflects a combination of capacitive (frequency-dependent region in high frequency) and resistive (frequency-independent region in low frequency) behaviors. The phase angle (Figure 1(c)) shows a gradual shift from -90 to 0 degrees while moving from high to low frequency. This further indicates a porous nature of coating and water penetration in the film on exposure to electrolyte [34].

3.2. Stage II: Two-Time-Constant Process. Typical Nyquist and Bode plots between 10 AND30 days of exposure in 5\% $\mathrm{NaCl}$ are shown in Figure 3. Almost two clearly resolved semicircles showing a two time constant process is seen from the Nyquist plot (Figure 3(a)) with an indication about the degradation of epoxy coating and an interaction between steel surface and solution [35]. One due to $C_{c}$ and $R_{\mathrm{po}}$ for the high frequency indicating the properties of coating and the other due to $C_{\mathrm{dl}}$ and $R_{t}$ for the low frequency reflecting the properties of the metal-electrolyte interaction or corrosion reaction at the metal surface. Figure 3 (b) shows the Bodemagnitude plot with two frequency-independent regions, and Figure 3(c) also confirms degradation of coating with the phase angle shifting from $-90^{\circ}$ degree at higher frequency towards 0 in the frequency range of $100 \mathrm{~Hz}$ showing coating characteristics which indicated the degradation of 


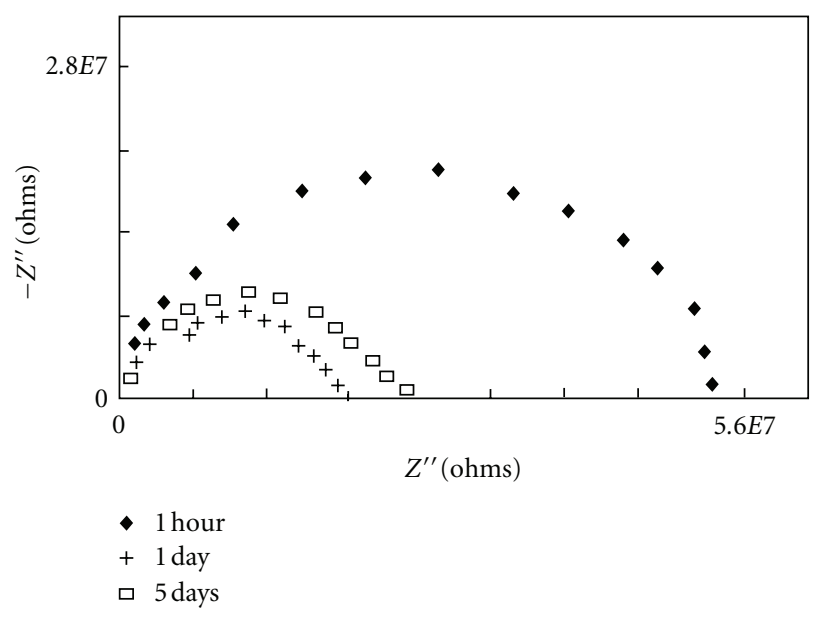

(a)

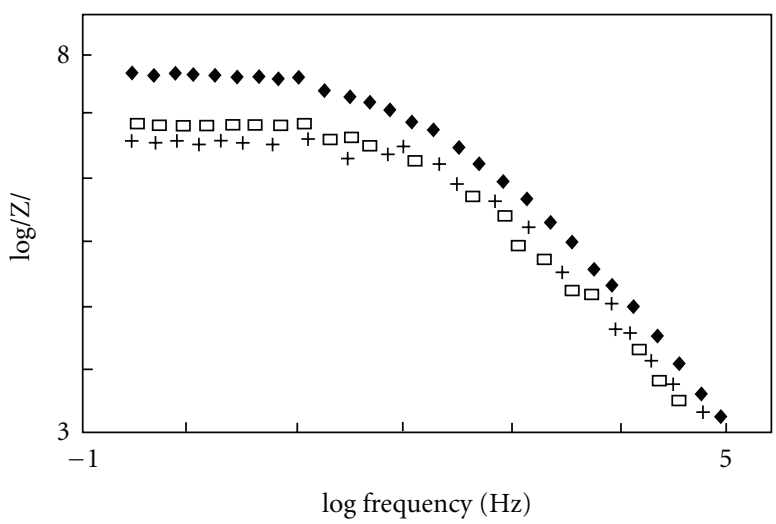

- 1 hour

+1 day

ㄷ 5 days

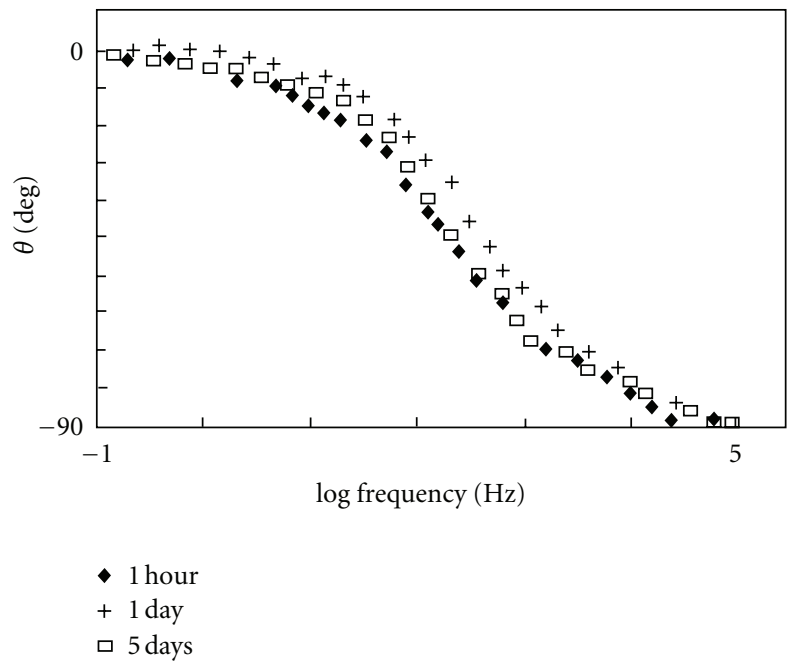

(c)

Figure 1: Typical (a) Nyquist, ((b) and (c)) Bode plot of EP primer coated steel in $5 \% \mathrm{NaCl}$ showing one time constant.

the coating. Further drop in phase angle and shifting towards 0 at $0.1 \mathrm{~Hz}$ is the consequence of charge transfer reaction and hence the second time constant. With the elapse of time the diagram shape changes and two capacitive semicircles can be observed, which, respectively, correlate to the electric properties of the organic coating and the faradic process at the substrate [37]. Conversely, a continuous decrease in the impedance values for the films is observed at all times. The equivalent electrical circuit as in Figure 2(b) can represent impedance response of this type.

Analysis of the impedance spectra in terms of the equivalent circuit Figure 2(b) allowed for the parameters $C_{c}$ and $R_{\mathrm{po}}$ to be determined. Changes in the magnitudes of these parameters as a function of their exposure time to the test solution are given in Table 2. The capacitance of the coating $C_{c}$ does not change significantly, and only a variation in $C_{c}$ values with longer time was found for the specimens exhibited by a significant delamination of the coating. Hence, analysis of $C_{c}$ values is not a sensitive procedure to establish the occurrence of delamination of coatings during shorter time of exposure.

The coating resistance values $R_{\mathrm{po}}$ of the coating against time of immersion are presented in Table 2. A continuous decrease of $R_{\text {po }}$ values with the elapsed time is clearly observed and is smaller than $10^{8} \mathrm{ohms}$ even shortly after immersion of the specimen in the test solution. This trend clearly indicates that the metal substrate is not much effectively isolated from the aggressive environment. Thus protection of the metal requires a thicker coating to be applied over than $200 \mu \mathrm{m}$. A similar observation was also reported with epoxy-polyamide with $100 \mu \mathrm{m}$ to $500 \mu \mathrm{m}$ coatings exposed in 3\% $\mathrm{NaCl}$ solution in earlier [37].

Visual observation on the 10th day showed the development of a brown spot at one place but no visual rupture of film or corrosion product. There has been varying opinion regarding the separation of two semicircles in the Nyquist 
TABLE 2: Equivalent circuit parameters of EPZ primer-coated steel in $5 \% \mathrm{NaCl}$.

\begin{tabular}{|c|c|c|c|c|c|c|}
\hline $\begin{array}{l}\text { Exposure duration } \\
\left({ }^{*} \text { hour/days }\right)\end{array}$ & $R_{\mathrm{po}}\left(10^{7}, \mathrm{ohms}\right)$ & $R_{t}\left(10^{6}, \mathrm{ohms}\right)$ & $C_{c}\left(10^{-9}\right.$, Faraday $)$ & $C_{\mathrm{dl}}\left(10^{-7}\right.$, Faraday $)$ & $R_{t} / R_{\text {po }}$ & $T_{m} / T_{f}$ \\
\hline$* 1$ & 5.6 & 5.26 & 8.50 & 1.096 & 0.094 & 1.21 \\
\hline 1 & 2.65 & 3.12 & 8.00 & 1.855 & 0.118 & 2.73 \\
\hline 5 & 2.14 & 1.92 & 7.10 & 3.016 & 0.089 & 3.81 \\
\hline 10 & 0.05 & 0.63 & 7.50 & 9.176 & 1.260 & 144.53 \\
\hline 15 & 0.204 & 1.35 & 7.60 & 4.289 & 0.662 & 36.19 \\
\hline 25 & 0.152 & 0.098 & 7.93 & 98.27 & 0.064 & 80.25 \\
\hline 30 & 0.082 & 0.062 & 9.00 & 936.90 & 0.076 & 830.00 \\
\hline 55 & 0.027 & 0.038 & 35.02 & 542.34 & 0.141 & 219.25 \\
\hline 80 & 0.0033 & 0.023 & 40.12 & 257.30 & 0.697 & 455.23 \\
\hline 100 & 0.0003 & 0.0058 & 43.00 & 996.50 & 1.933 & 4446.15 \\
\hline
\end{tabular}

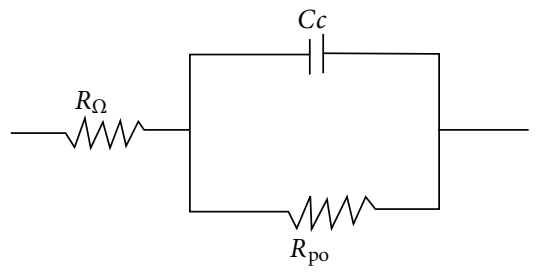

(a)

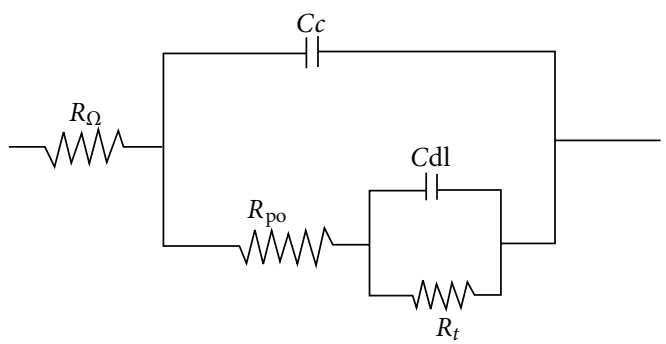

(b)

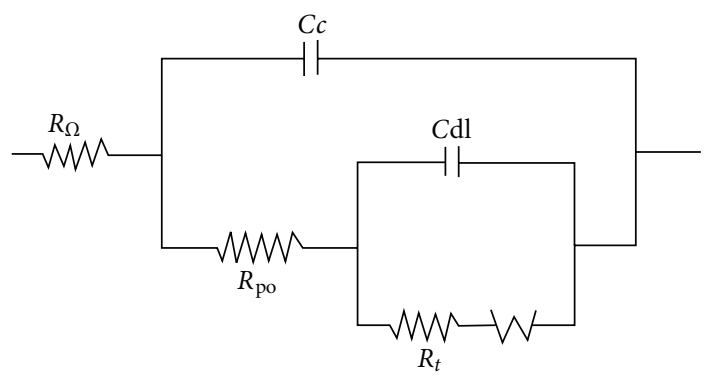

(c)

FIGURE 2: Equivalent electrical circuit representing coated metalelectrolyte interface. (a) Intact coating, (b) without and (c) with Warburg impedance.

plot $[34,38]$. The complex plane plot obtained in this study is made up of two semicircles with time constants (units in seconds) for the paint film $T_{f}$ and for metal $T_{m}$ as given by:

$$
T_{f}=C_{c} \cdot R_{\mathrm{po}}, \quad T_{m}=R_{t} C_{\mathrm{dl}}
$$

The circuit parameters and the time constants calculated during the period of 10-30 days (stage II) are given in Table 2. They are in almost in agreement with the criteria for the separation of two semicircles reviewed by Ramesh et al. [29] as follows:

$$
0.2 \leq\left(\frac{R_{t}}{R_{\text {po }}}\right) \leq 5 ; \quad \frac{T_{m}}{T_{f}} \geq 20 .
$$

Further in this system, the $T_{m}$ was much higher than $T_{f}$ $\left(T_{m} \gg>T_{f}\right)$, which had a condition given for observation of two distinguished semicircle in the Nyquist plot [39]. The circuit parameters were estimated using the equivalent circuit model as given in Figure 1(b). Thus, the second semicircle undoubtedly represented the charge transfer reaction at coating-metal interface [35].

3.3. Stage III: With Warburg Impedance. Second semicircle of the Nyquist plot shown in Figure 3(a) gradually changed to a tail with an angle of inclination to real axis but the first semicircle still continues after 30 days. During this period 2 to 3 small brown spots developed and in the initial spot the coating was damaged and corrosion was visible along the periphery of damaged coating. Thereafter, the floating of rust particles was seen in the electrolytic solution. Representative Nyquist and Bode plots for 80 and 100 days are shown in Figure 4 . The Nyquist plot shows a high frequency semicircle and a tail in the low frequency with an angle of inclination of nearly $45^{\circ}$ at lower frequency. The Bode-phase plot (Figure 4(c)) shows limited capacitive characteristic but frequency independent impedance is seen in the low frequency range. It can be seen that the phase angle shifts towards $-40^{\circ}$ in the corresponding low frequency range. This type of characteristics pertains to the diffusion controlled process with mass transfer $[34,39,40]$ and can be modeled in terms of equivalent circuit as in Figure 2(c) shows the Randles circuit. The elements used in this EC, an excessive with $W$, Warburg impedance, that is given by [35]

$$
W=[\sigma \omega]^{-1 / 2}(1-j),
$$

where $j=$ imaginary number, $\sigma=$ Warburg coefficient, $\omega=$ $2 \pi f$ is the angular frequency, and $f=$ frequency. 

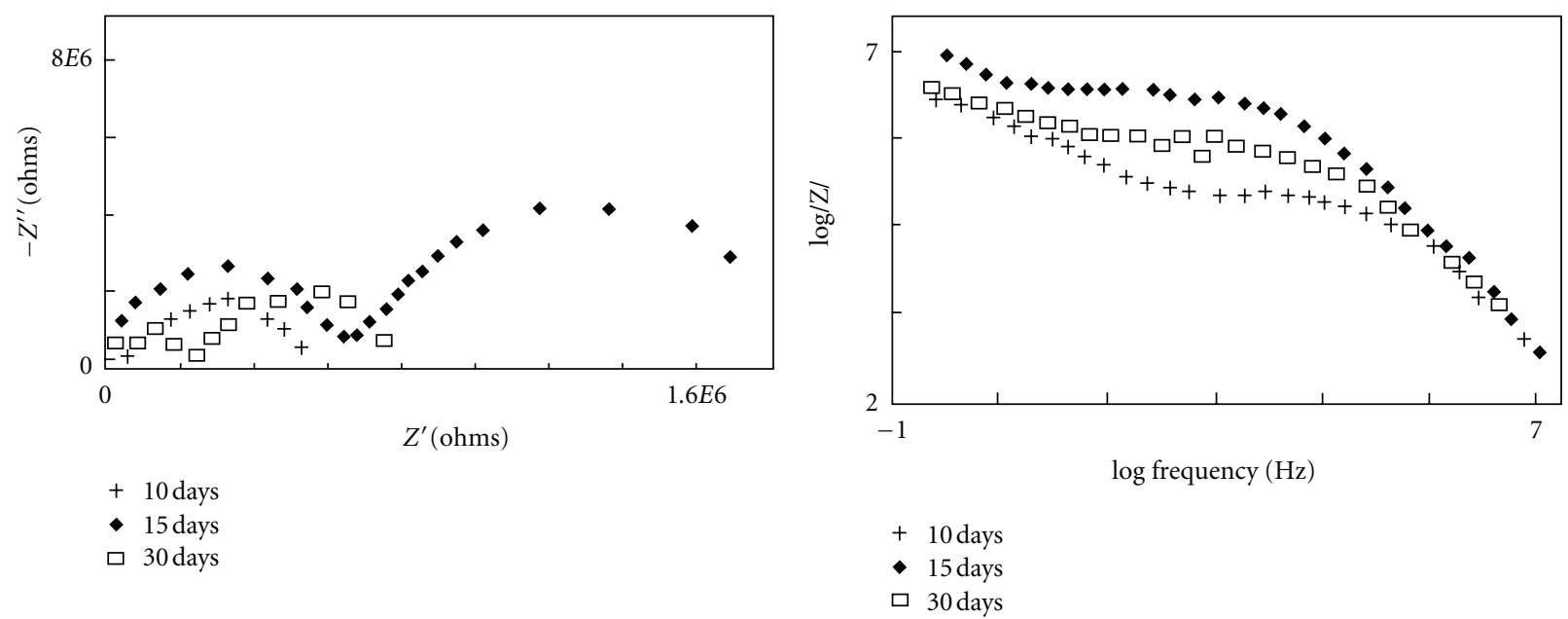

(a)

(b)

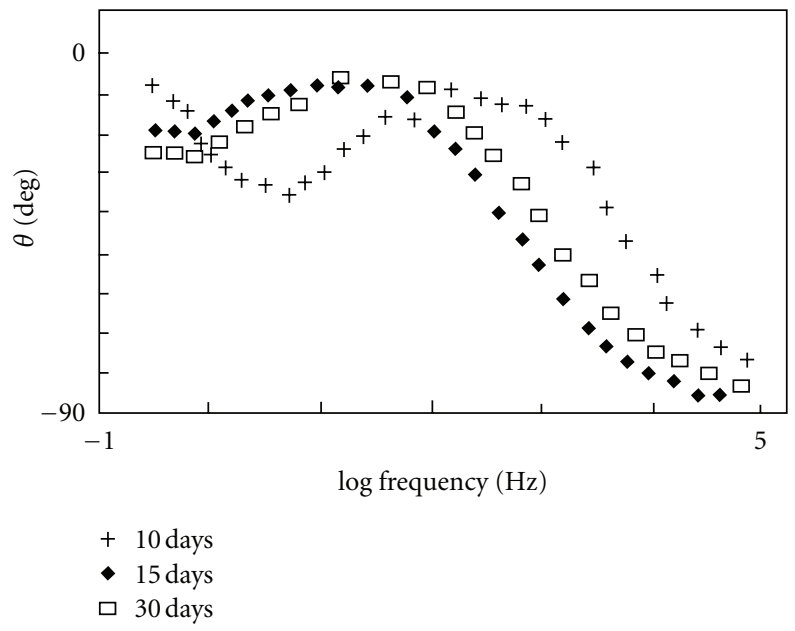

(c)

Figure 3: (a) Nyquist ((b) and (c)) and Bode plots for EPZ primer-coated steel in $5 \% \mathrm{NaCl}$ showing two time constants.

The Randless circuit is used when the corrosion is controlled by a diffusion process with diffusion of reactant and/ or products to the interface. For diffusion-controlled process the impedance is given by [41],

$$
Z_{o}=\frac{\left[\tan B(j \omega)^{1 / 2}\right]}{Y_{o}(j \omega)^{1 / 2}},
$$

where $B=l /(D)^{1 / 2}-D$ is the diffusion coefficient, $l$ is the diffusion layer thickness, and $Y_{o}=\left(\sigma(2)^{1 / 2}\right)^{-1}$.

The variation of $C_{c}, R_{\mathrm{po}}$, and $R_{t}$ of EPZ coating during different period of exposure is shown in Figure 5. The $C_{c}$ nearly remains unchanged up to 25 days after a slight initial fluctuation up to 5 days. It suddenly increases after 30 days, which reflects a deterioration of coating. The other parameter $R_{t}$ gives an indication about corrosion process at steel surface. This can be used to study the effectiveness of coatings in the protection of metals. Figure 5 also reflects the changes of $R_{t}$ values slowly during initial exposure and attains a minimum after 10 days and further increases up to
15 days. Thereafter, it slowly decreases until 30 days followed by decreases as fasts about 100 days of exposure due to the penetration of solution between the coating and steel surface. This penetration can be passed through breakdown sites of the coating [28]. The $R_{t}$ increases between 10 and 15 days of exposure indicating the protection of steel by the formation of corrosion products, and thereafter it decreases by penetration of electrolyte. After 30 days of exposure, appearance of a depressed semicircle tail in the Nyquist plot indicates the presence of Warburg-type impedance- and a sharp increase in $C_{c}$ beyond this period indicates the deterioration of primer coating. It was not possible to estimate $R_{t}$ beyond 30 days, which was masked by low-frequency tail or due to severely interacting time constants $[37,41,42]$.

3.4. Break Point Frequency. Degradation of the coated specimens was first investigated by means of the break-point frequency method proposed by Haruyama et al. [19]. In this method, values of a characteristic breakdown frequency $f_{b}$ are determined experimentally from the impedance spectra as they are related to the delaminated area in the initial stage 


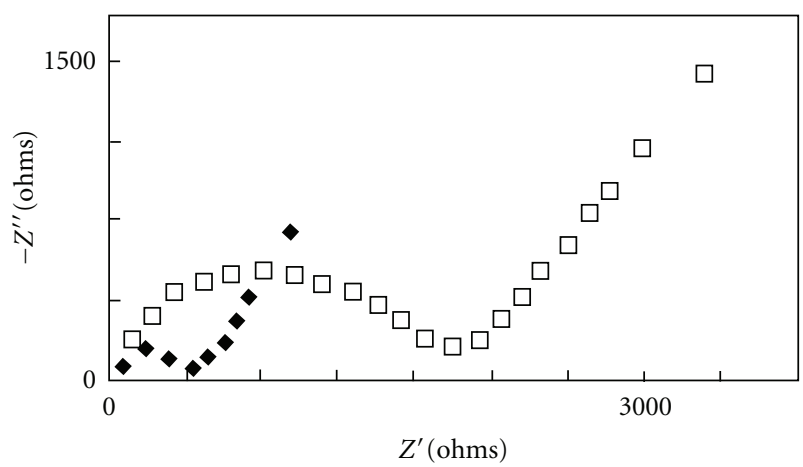

$\square 80$ days

- 100 days

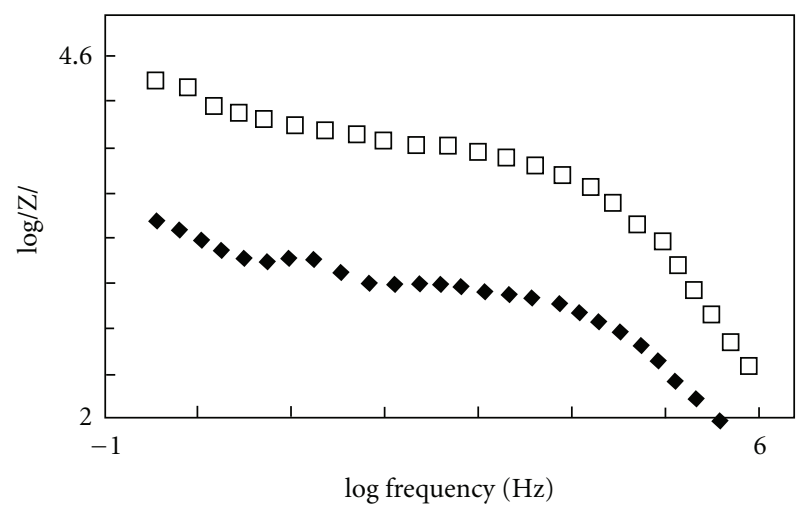

$\square 80$ days

- 100 days

(a) (b)

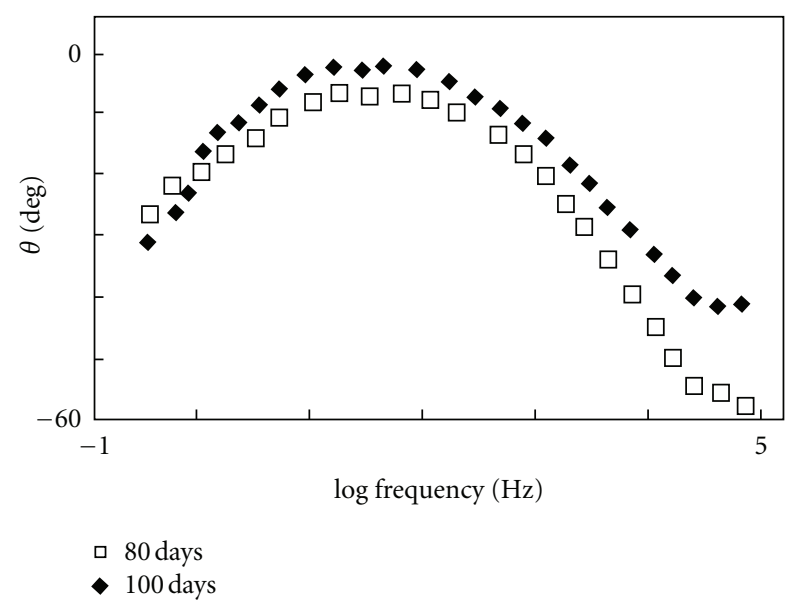

(c)

Figure 4: (a) Nyquist and ((b) and (c)) Bode plots of EPZ primer coated steel in 5\% $\mathrm{NaCl}$ showing diffusion process.

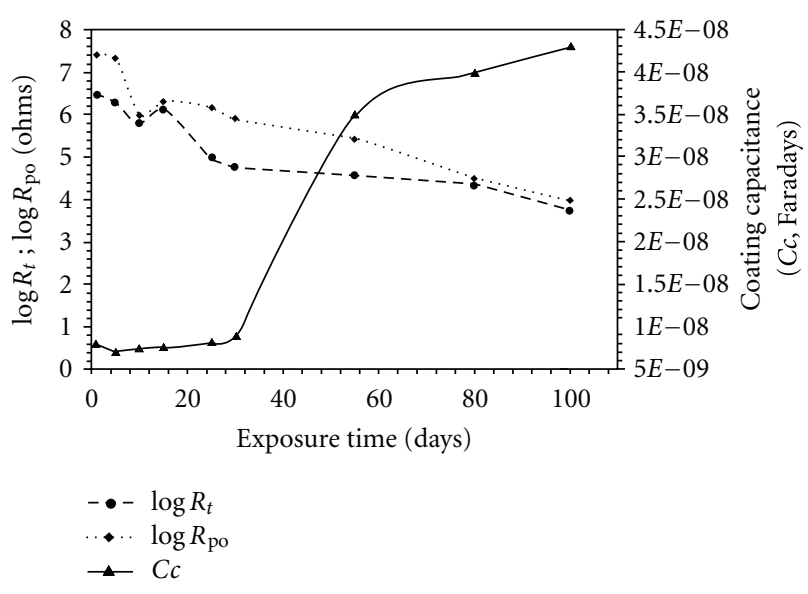

Figure 5: Time dependence of $C_{c}, R_{\mathrm{po}} / R_{t}$ of EPZ coated steel in $5 \%$ $\mathrm{NaCl}$.

of delamination. This characteristic breakdown frequency $f_{b}$ is the frequency corresponding to $45^{\circ}$ phase angle in capacitive-resistive region which has also been used by Scully et al. [22-24] and Mansfeld [41] to study the electrochemically active area of coated metal in corrosive environment.

The time dependence of $f_{b}$ is quite sensitive method to the development of localized low-resistive defects, delamination, and also actively corroding areas, and hence it is a good predictive parameter for long-term coating performance. The variation of $f_{b}$ for EPZ primer-coated steel is plotted against the time of immersion and is shown in Figure 6. Since the break-point frequency is related to the delaminated area [19]. The $f_{b}$ shows minor changes between 1 and 5 days and then suddenly shifts to higher frequency at 10 days followed by a reverse at 15 days. Its shift to high frequency is prominent after 30 days. The shift of $f_{b}$ to higher values with increased exposure duration was interpreted as an increase due to the delaminated area or on the thickness of the coated film [37]. The shift of $f_{b}$ to low frequency was followed by an increase in $R_{\mathrm{po}}$ and to high frequency by a decrease in $R_{\mathrm{po}}$. The improvement in the coating behavior and the delamination was clearly reflected in terms of shift of $f_{b}$ to low or high frequencies, respectively $[22,23]$. The visual observation of a brown spot on the 10th day in the coating 


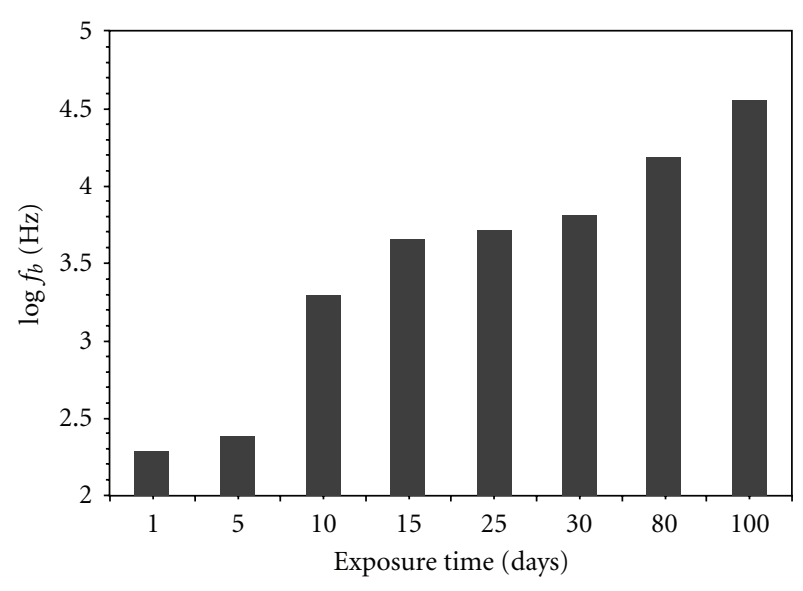

Figure 6: Time dependence of break-point frequency $\left(f_{b}\right)$ of EPZ coated steel in $5 \% \mathrm{NaCl}$.

was reflected in the impedance behavior in terms of decrease in $R_{\mathrm{po}}$, appearance of $R_{t}$ (second semicircle), and sudden drop in $E_{\mathrm{oc}}$ to more negative value shown in Figure 7. This was possible due to the creation of local defect in the coating on exposure to electrolyte. But the spreading of damage was restricted due to the presence of zinc phosphate anticorrosive pigment, which passivates the metal, and blocking of microspores of the primer coating takes place [43]. This was further supported by an increase in $R_{t}$ and $R_{\mathrm{po}}$ between 10 and 15 days. Further a slower rate of damage of coating takes place up to 30 days followed by delamination beyond it. During this period, the shift of $f_{b}$ to higher frequency indicates an increase in the corroding area. The passivation effect was limited due to the low concentration of pigment used and high chloride ion concentration of electrolyte [44].

Trend in Figure 6 qualitatively represents the time course of degradation of the coated material. It is seen in Figure 6 that the rate of delamination of the coated specimen increases steeply after 30 days for the remaining exposure time. At that stage, the occurrence of delamination could be discriminated by visual observation. The values of the break-point frequencies are also found to vary little with time, showing a characteristic small increase at early exposure times which is followed by a slow decay to reach a rather stationary value at sufficiently longer exposure times. This behavior is characteristic for a coated system which effectively protects the metal substrates from the aggressive environment [37].

Many workers have stressed the proper analysis of the Nyquist plots. Alternative fitting procedure of curves, constant phase element $[27,45]$, and most probable equivalent circuit concept have been developed and used for accurate determination of impedance parameters which was used for predicting long-term performance taking into account the complex nature of impedance curves involved in different systems. In this study, impedance parameters could be determined using typical equivalent circuit as in Figure 2 for different stages of degradation. It is important to mention that the visual observation of coatings (on set of degradation,

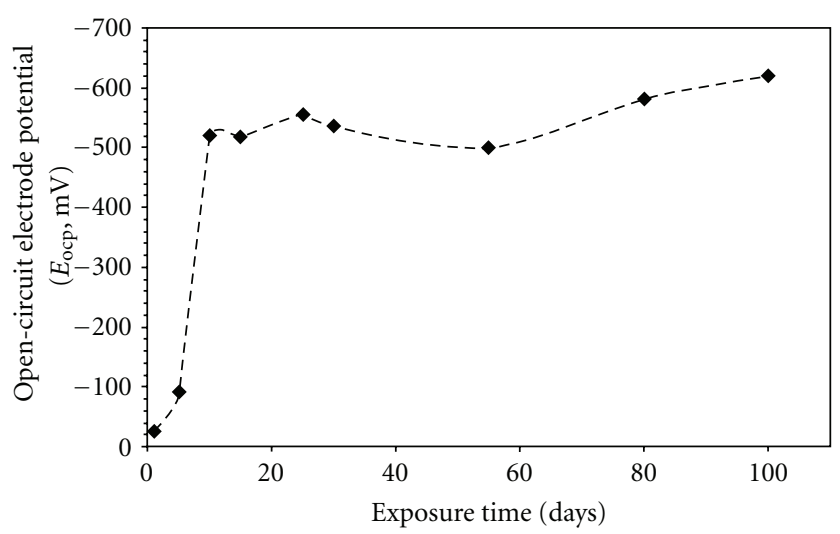

Figure 7: Time dependence of $E_{\text {oc }}$ of EPZ-coated steel in $5 \% \mathrm{NaCl}$.

corrosion) and corresponding shapes of the Nyquist plots were showing close resemblance.

Duplicate sets (triplicates) of experiments were carried out. The similar three stages (namely, one time constant, two time constant, and diffusion controlled) of degradation, and initial decrease and further increased were also visualized. However, the exact exposure period during which these stages appeared was varying. This is possible because of the variation of coating thickness, uniformity of pigments, and crosslinking of epoxy polymer coating. Further the degradation mechanism of the polymer coating is dependent on the metal characteristics, the application limitations, polymer properties, and aggressive environment of exposure. Conventional spraying-coated panels used in this study and no further finishing touches were given keeping in view the practical conditions. Hence it will not be worth to index this as the performance of epoxy primer in absolute terms, rather a reflection of degradation pattern under a specified condition. Nevertheless, the experimental results, in fact, represented typical simulated curves [34] for different equivalent circuit for coated metals (intact and deteriorating coatings). Thus, EIS seems to be an ideal technique for analysis of the mode of deterioration/degradation of polymer coating and the corrosion of coated steel.

The $R_{\mathrm{ct}}$ and $C_{\mathrm{dl}}$ are the two parameters which are normally used to specify the disbanding of the coatings and the onset of corrosion at the interface. These parameters decrease the $R_{\mathrm{ct}}$ and increase the capacitances during the first few days, indicating the entry of electrolyte into the epoxy coating [46]. The $C_{\mathrm{dl}}$ is a measure of an area from which the coatings have been disbanded and can be measured only at a very advanced stage of deterioration. The change in $C_{\mathrm{dl}}$ values may depend either on disbanding of the coating or on the accumulation of corrosion products during the corrosion processes [47].

In the present work, the $C_{\mathrm{dl}}$ value increase (shown in Table 2) upon longer duration implies the disbanding of coatings observed in visual observation. Variation in capacitance with immersion time could be induced with water uptake in coatings. The penetration of $\mathrm{Na}^{+}$and $\mathrm{Cl}^{-}$ions in the coatings can also influence the capacitance [48]. 
3.5. Potential-Time Measurement. Electrode potential $\left(E_{\mathrm{oc}}\right)$ against exposure time provides a means of estimating the corrosion tendency of specimens and also supports the EIS study about the degradation of EPZ coating of this study. Figure 7 represents the $E_{\mathrm{oc}}$, of EPZ-coated specimens, plotted against immersion time. It is clearly marked that the EPZ hybrid coating exist minimum potential as less negative during initial period of exposure (i.e., after one day immersion shows $-26 \mathrm{mV}$ versus SCE) due to anodic passive effect [49]. Further a sudden drop between 1 day and 10 days from $-26 \mathrm{mV}$ to $-539 \mathrm{mV}$ versus SCE, any negative shift of $E_{\mathrm{oc}}$, is explained as due to permeation of moisture or water through the pores of the films and initiates active corrosion in the metal film interface [50]. In longer duration the same formulation of coating material which had more negative potential shift from -539 to $-620 \mathrm{mV}$ versus SCE takes place, which may be owing to the fluctuation of anodic and cathodic sites.

\section{Conclusions}

Epoxy coating degradation was observed up to 100 days of exposure in corrosive $5 \% \mathrm{NaCl}$ environment at room temperature. Well-controlled EIS experiments of zinc phosphate-pigmented coating can provide the detailed mechanistic information on the degradation behavior on coated steel substrate. Three distinct stages investigated in the degradation behavior of a zinc phosphate-pigmented epoxy primer have been presented in this study. Visual observation of deterioration of primer coating was also reflected in the shapes of the Nyquist and the Bode plots. The corrosion of substrate by the ingress of ionic species through coating increases disbanding between coating and substrate, which promotes the degradation of coating by the action of aggressive chemical $(5 \% \mathrm{NaCl})$ environment.

The passivation effect due to anticorrosive pigment could be the reason for the improvement in coating characteristics and diminish the porosity on early stage of exposure but during longer exposure enhances the porosity and undergoes the penetration of electrolyte through the coating causing the deterioration of coating. Coating thickness of the material coated became thinner; porous nature of the films and high concentration of $\mathrm{Cl}^{-}$ion being responsible for breakdown of the coating during the course of longer exposure in aggressive chemical environment was well established and also supported throughout this study. Thus, results of the present study indicate that the zinc phosphate-containing epoxypolyamide coatings had good corrosion resistance, which could be an eco-friendly alternate to the traditional conversion coatings.

\section{Acknowledgment}

D. Ramesh expresses thanks to his wife Mrs. V. Vidyadevi Ramesh, for constant encouragement and heartfelt support for making this research paper and for making many insightful suggestions for its improvement.

\section{References}

[1] N. Kouloumbi, G. M. Tsangaris, and S. T. Kyvelidis, "Evaluation of corrosion behavior of metal-filled polymeric coatings," Journal of Coatings Technology, vol. 66, no. 839, pp. 83-88, 1994.

[2] H. Leidheiser, "Towards a better understanding of corrosion beneath organiccoatings," Corrosion, vol. 39, no. 5, pp. 189201, 1983.

[3] F. Deflorian, S. Rossi, P. Kamarchik, L. Fedrizzi, and P. L. Bonora, "Degradation mechanism of electrodeposited coatings in alkaline solution," Progress in Organic Coatings, vol. 47, no. 2, pp. 103-111, 2003.

[4] P. Li, T. C. Tan, and J. Y. Lee, "Corrosion protection of mild steel by electroactive polyaniline," Synthetic Metals, vol. 88, no. 3, pp. 237-242, 1997.

[5] D. M. Lenz, M. Delamar, and C. A. Ferreir, "Improvement of the anticorrosion properties of polypyrrole by zinc phosphate pigment incorporation," Progress in Organic Coatings, vol. 58, no. 1, pp. 64-67, 2007.

[6] D. Ramesh and T. Vasudevan, "Synthesis and physicochemical evaluation of water," Progress in Organic Coatings, vol. 66, no. 2, pp. 93-98, 2009.

[7] X. Liu, J. Xiong, Y. Lv, and Y. Zuo, "Study on corrosion electrochemical behavior of several different coating systems by EIS," Progress in Organic Coatings, vol. 64, no. 4, pp. 497-503, 2009.

[8] K. Ramesh, S. Ramesh, and A. K. Arof, "Study on the properties of the hybrid organic coatings for corrosion protection," International Journal of Fundamental Physical Sciences, vol. 1, no. 2, pp. 34-36, 2011.

[9] R. Pang, Y. Zuo, Y.-M. Tang, and J.-P. Xiong, "Electrochemical impedance spectroscopy study of failure process of an epoxy/fluorocarbon coating system," Acta Physico-Chimica Sinica, vol. 28, no. 5, pp. 1146-1152, 2012.

[10] F. Deflorian, L. Fedrizzi, S. Rossi, F. Buratti, and P. L. Bonora, Progress in Organic Coatings, vol. 41, p. 93, 2001.

[11] J. R. Scully and S. T. Hensley, "Lifetime prediction for organic coatings on steel and a magnesium alloy using electrochemical impedance methods," Corrosion, vol. 50, no. 9, pp. 705-716, 1994.

[12] E. P. M. van Westing, G. M. Ferrari, and J. H. W. de Wit, "The determination of coating performance with impedance measurements-III. In situ determination of loss of adhesion," Corrosion Science, vol. 36, no. 6, pp. 979-994, 1994.

[13] E. P. M. van Westing, G. M. Ferrari, and J. H. W. de Wit, "The determination of coating performance with impedance measurements-I. Coating polymer properties," Corrosion Science, vol. 34, no. 9, pp. 1511-1530, 1993.

[14] G. Grundmeier, W. Schmidt, and M. Stratmann, "Corrosion protection by organic coatings: electrochemical mechanism and novel methods of investigation," Electrochimica Acta, vol. 45, no. 15-16, pp. 2515-2533, 2000.

[15] L. V. S. Philippe, S. B. Lyon, C. Sammon, and J. Yarwood, "Validation of electrochemical impedance measurements for water sorption into epoxy coatings using gravimetry and infra-red spectroscopy," Corrosion Science, vol. 50, no. 3, pp. 887-896, 2008.

[16] D. Raps, T. Hack, J. Wehr et al., "Electrochemical study of inhibitor-containing organic-inorganic hybrid coatings on AA2024," Corrosion Science, vol. 51, no. 5, pp. 1012-1021, 2009.

[17] N. Pebere, T. Picaud, M. Duprat, and F. Dabosi, "Evaluation of corrosion performance of coated steel by the impedance 
technique," Corrosion Science, vol. 29, no. 9, pp. 1073-1086, 1989.

[18] E. Spengler, F. L. Fragata, I. C. P. Margarit, and O. R. Mattos, "Corrosion protection of low toxicity paints," Progress in Organic Coatings, vol. 30, no. 1, pp. 51-57, 1997.

[19] S. Haruyama, M. Asari, and T. Tsuru, "Mass transport in blistering of coating steel," in Proceedings of the Symposium on Corrosion Protection by Organic Coatings, M. Kendig and H. Leihaiser Jr., Eds., vol. 87-2, Electrochemical Society ECS, Pennington, NJ, USA, 1987.

[20] G. P. Bierwagen, L. He, J. Li, L. Ellingson, and D. E. Tallman, "Studies of a new accelerated evaluation method for coating corrosion resistance-thermal cycling testing," Progress in Organic Coatings, vol. 39, no. 1, pp. 67-78, 2000.

[21] M. C. S. S. Macedo, I. C. P. Margarit-Mattos, F. L. Fragata, J. B. Jorcin, N. Pébère, and O. R. Mattos, "Contribution to a better understanding of different behaviour patterns observed with organic coatings evaluated by electrochemical impedance spectroscopy," Corrosion Science, vol. 51, no. 6, pp. 1322-1327, 2009.

[22] John R. Scully, "Electrochemical impedance of organic-coated steel: correlation of impedance parameters with long-term coating deterioration," Journal of the Electrochemical Society, vol. 136, no. 4, pp. 979-990, 1989.

[23] J. R. Scully and H. P. Hack, "Defect area determination of organic coated steels in seawater using the breakpoint frequency method," Journal of the Electrochemical Society, vol. 138, no. 1, pp. 33-40, 1991.

[24] J. Scully and M. Kendig, "Basic aspects of electrochemical impedance application for the life prediction of organic coatings on metals," Corrosion, vol. 46, no. 1, pp. 22-29, 1990.

[25] F. Mansfeld, S. L. Jeanjaquet, and M. W. Kendig, "An electrochemical impedance spectroscopy study of reactions at the metal/coating interface," Corrosion Science, vol. 26, no. 9, pp. 735-742, 1986.

[26] B. J. Hepburn, K. R. Gravers, and T. D. Scantlebury, British Corrosion Journal, vol. 21, p. 105, 1986.

[27] F. M. Geenen, J. H. W. De Wit, and E. P. M. Van Westing, "An impedance spectroscopy study of the degradation mechanism for a model epoxy coating on mild steel," Progress in Organic Coatings, vol. 18, no. 3, pp. 299-312, 1990.

[28] S. Masadeh, Journal of Minerals \& Materials Characterization \& Engineering, vol. 4, no. 2, pp. 75-84, 2005.

[29] D. Ramesh, P. Shakkthivel, A. Susaimanickam, A. Kalpana, and T. Vasudevan, Corrosion Science and Technology, vol. 5, no. 2, pp. 62-68, 2006.

[30] K. Kamaraj, S. Sathiyanarayanan, and G. Venkatachari, "Electropolymerised polyaniline films on AA 7075 alloy and its corrosion protection performance," Progress in Organic Coatings, vol. 64, no. 1, pp. 67-73, 2009.

[31] S. Skale, V. Doleček, and M. Slemnik, "Electrochemical impedance studies of corrosion protected surfaces covered by epoxy polyamide coating systems," Progress in Organic Coatings, vol. 62, no. 4, pp. 387-392, 2008.

[32] S. Chaudhari, S. R. Sainkar, and P. P. Patil, "Poly(o-ethylaniline) coatings for stainless steel protection," Progress in Organic Coatings, vol. 58, no. 1, pp. 54-63, 2007.

[33] D. Shikha, P. K. Kamani, and M. C. Shukla, "Studies on synthesis of water-borne epoxy ester based on RBO fatty acids," Progress in Organic Coatings, vol. 47, no. 2, pp. 87-94, 2003.

[34] G. W. Walter and, Corrosion Science, vol. 26, p. 22, 1986.

[35] A. M. Nagiub, "Evaluation of corrosion behavior of copper in chloride media using electrochemical impedance spectroscopy
(EIS)," Portugaliae Electrochimica Acta, vol. 23, pp. 301-314, 2005.

[36] D. F. Wei, I. Chatterjee, and D. A. Jones, Corrosionp, vol. 52, p. 417, 1996.

[37] S. González, M. A. Gil, J. O. Hernández, V. Fox, and R. M. Souto, "Resistance to corrosion of galvanized steel covered with an epoxy-polyamide primer coating," Progress in Organic Coatings, vol. 41, no. 1-3, pp. 167-170, 2001.

[38] A. M. Fenelon and C. B. Breslin, "An electrochemical study of the formation of benzotriazole surface films on copper, zinc and a copper-zinc alloy," Journal of Applied Electrochemistry, vol. 31, no. 5, pp. 509-516, 2001.

[39] F. El-Taib Heakal and S. Haruyama, "Impedance studies of the inhibitive effect of benzotriazole on the corrosion of copper in sodium chloride medium," Corrosion Science, vol. 20, no. 7, pp. 887-898, 1980.

[40] D. O. Mac Donald, Impedance Spectroscopy, John Wiley \& Sons, 1987.

[41] F. Mansfeld, "Discussion of "determination of the reactive area of organic coated metals: physical meaning and limits of the break-point method", a comment on the paper by F. Deflorian et al." Electrochimica Acta, vol. 39, no. 10, pp. 1451-1452, 1994.

[42] K.-M. Yin and L. I. Lu, "Parametric study on the electrochemical impedance spectroscopy of organic-coated steels in hydrochloric acid solutions," Journal of Coatings Technology, vol. 75, no. 941, pp. 65-72, 2003.

[43] S. C. J. Atwood, Oil and Colour Chemists' Association, vol. 4, p. 128, 1992.

[44] D. Ramesh, Development of water-based paints and their evaluation [Ph.D. thesis], Alagappa University, Karaikudi, India, 2004.

[45] E. P. M. van Westing, G. M. Ferrari, and J. H. W. De Wit, “The determination of coating performance using electrochemical impedance spectroscopy," Electrochimica Acta, vol. 39, no. 7, pp. 899-910, 1994.

[46] D. M. Dražić and Z. Kačarević-Popović, "The sorption characteristics of epoxy coatings electrodeposited on steel during exposure to different corrosive agents," Corrosion Science, vol. 38, no. 9, pp. 1513-1523, 1996.

[47] J. J. Suay, M. T. Rodríguez, K. A. Razzaq, J. J. Carpio, and J. J. Saura, "The evaluation of anticorrosive automotive epoxy coatings by means of electrochemical impedance spectroscopy," Progress in Organic Coatings, vol. 46, no. 2, pp. 121129, 2003.

[48] C. Lepen et al., Progress in Organic Coatings, vol. 46, p. 77, 2003.

[49] S. G. Real, A. C. Elias, J. R. Vilche, C. A. Gervasi, and A. Di Sarli, "An electrochemical impedance spectroscopy study of zinc rich paints on steels in artificial sea water by a transmission line model," Electrochimica Acta, vol. 38, no. 14, pp. 2029-2035, 1993.

[50] O. Ferraz, E. Cavalcanti, and A. R. Di Sarli, "The characterization of protective properties for some naval steel/polimeric coating $/ 3 \% \mathrm{NaCl}$ solution systems by EIS and visual assessment," Corrosion Science, vol. 37, no. 8, pp. 1267-1280, 1995. 

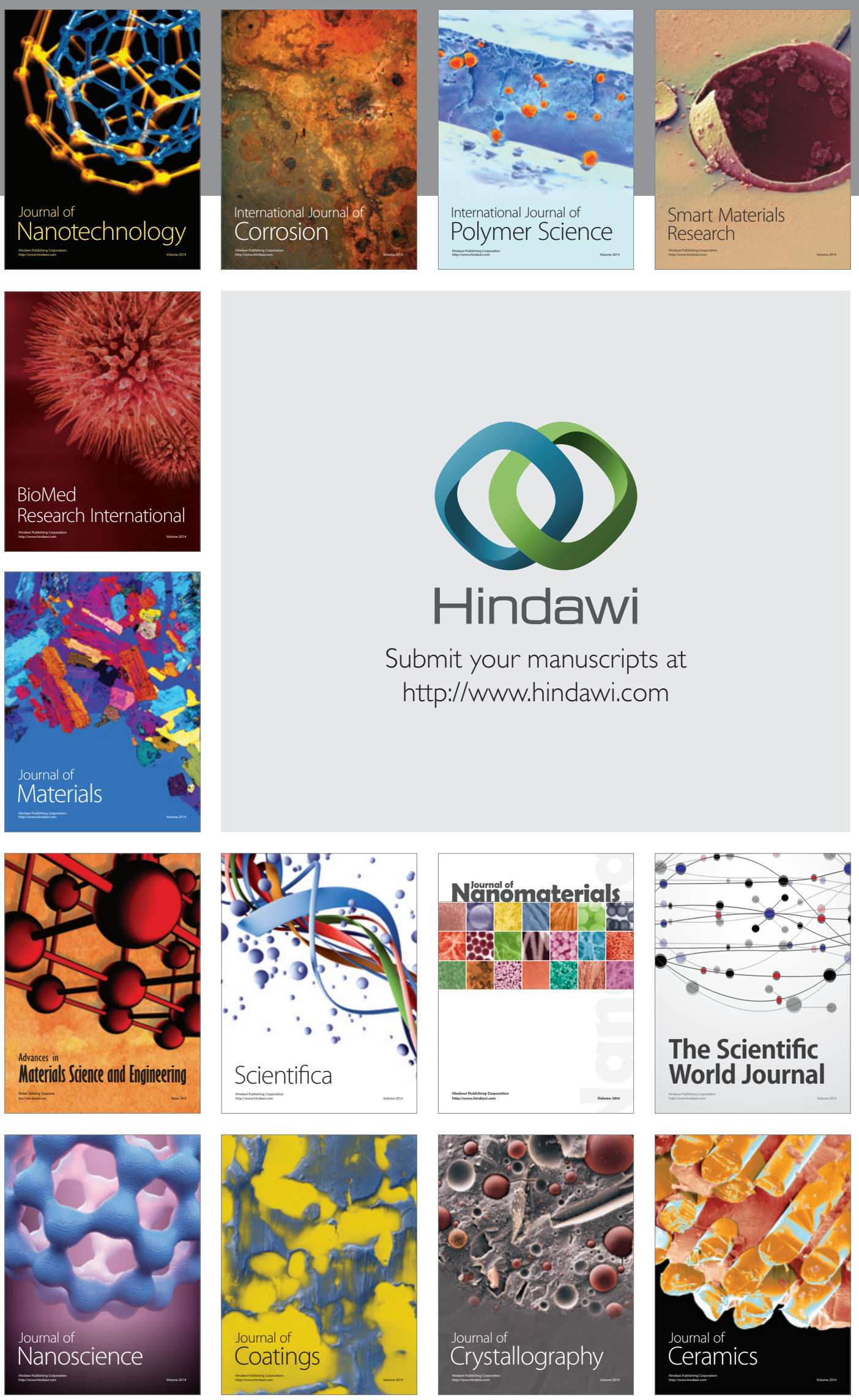

The Scientific World Journal

Submit your manuscripts at

http://www.hindawi.com

\section{World Journal}

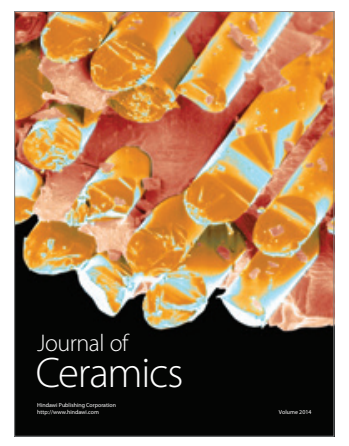

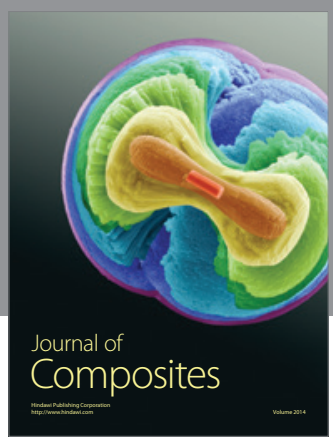
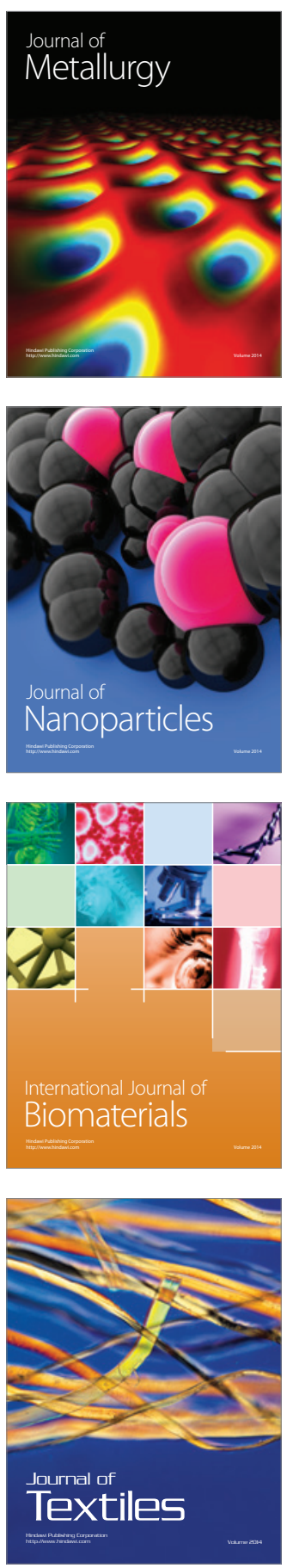